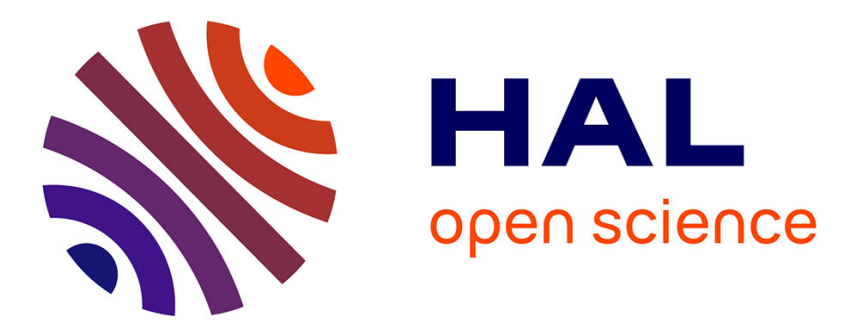

\title{
Commande non linéaire en vitesse d'un servomoteur synchrone avec calcul de trajectoire et estimation du couple résistant
}

\author{
Bruno Le Pioufle, G. Georgiou
}

\section{To cite this version:}

Bruno Le Pioufle, G. Georgiou. Commande non linéaire en vitesse d'un servomoteur synchrone avec calcul de trajectoire et estimation du couple résistant. Journal de Physique III, 1992, 2 (10), pp.19051924. 10.1051/jp3:1992221 . jpa-00248854

\section{HAL Id: jpa-00248854 https://hal.science/jpa-00248854}

Submitted on 1 Jan 1992

HAL is a multi-disciplinary open access archive for the deposit and dissemination of scientific research documents, whether they are published or not. The documents may come from teaching and research institutions in France or abroad, or from public or private research centers.
L'archive ouverte pluridisciplinaire HAL, est destinée au dépôt et à la diffusion de documents scientifiques de niveau recherche, publiés ou non, émanant des établissements d'enseignement et de recherche français ou étrangers, des laboratoires publics ou privés. 


\title{
Commande non linéaire en vitesse d'un servomoteur synchrone avec calcul de trajectoire et estimation du couple résistant
}

\author{
B. Le Pioufle $\left({ }^{1}\right)$ et G. Georgiou $\left({ }^{2}\right)$ \\ (1) Laboratoire d'Electricité, Signaux et Robotique, U.A. C.N.R.S. D1375, 61 av. Pt. Wilson, \\ 94230 Cachan, France \\ (2) Laboratoire de Signaux et Systèmes, U.M. C.N.R.S. 14, Plateau du Moulon, 91192 Gif-sur- \\ Yvette, France
}

(Reçu le 21 novembre 1991, révisé le 10 juin 1992, accepté le 29 juin 1992)

\begin{abstract}
Résumé. - Dans cet article, une méthode est proposée pour améliorer robustesse et performances de la commande non linéaire du servomoteur synchrone. La méthode consiste à calculer en temps réel une trajectoire asymptotique pour la vitesse, engendrant un couple moteur optimum pendant la durée du transitoire de vitesse. Un estimateur du couple résistant est utilisé. Des simulations et des résultats expérimentaux sont présentés.

Abstract. - In this paper, a method improving performances and robustness of nonlinear control of synchronous servomotor is proposed. A speed trajectory, asymptotically tracked by the nonlinear controller, is computed in real time and permits to obtain the optimum torque during the speed transients. A load torque estimator is included in the control. Simulation and experimentation results are presented.
\end{abstract}

\section{Introduction.}

Dans les applications de robotique où la vitesse variable s'avère nécessaire, les machines à courant continu furent les premières à faire leur apparition sur le marché industriel. La simplicité de la commande en vitesse de ces machines se conjugue à une extrême simplicité du variateur de vitesse associé. Avec l'essor du microprocesseur et de la micro-informatique, les équipes de recherche synthétisèrent des commandes numériques plus performantes que les simples régulateurs P.I.D. usuels [1], et des travaux pour modéliser finement le fonctionnement des convertisseurs de puissance associés firent leur apparition.

Avec le développement de l'électronique de puissance commencèrent à apparaître les commandes à vitesse variable de machines à courants alternatifs. Les moteurs à courants alternatifs présentent l'avantage d'avoir de meilleures performances (notamment en terme de couple massique [2]), et de ne pas avoir de collecteur mécanique (ce collecteur pose des problèmes d'entretien et de comportement dans les environnements difficiles). En revanche, ces moteurs sont plus exigeants. En effet, le moteur à courant continu est alimenté par un convertisseur statique simple, alors que pour les machines à courants alternatifs, la fonction 
collecteur est réalisée par un ensemble électronique : un onduleur de puissance, un capteur de position (dans la mesure du possible), et une commande dont le degré de sophistication est lié aux performances escomptées par le variateur [3].

Une approximation usuellement utilisée dans la commande de machine, consiste à considérer que les dynamiques de la vitesse et des courants sont suffisamment éloignées pour pouvoir contrôler ces variables de façon découplée dans des boucles de régulation imbriquées [4-9]. La structure de commande en boucles imbriquées consiste à asservir le couple, puis la vitesse en prenant comme référence de couple l'erreur corrigée en vitesse.

Des études [10] montrent les conséquences qu'une telle approximation peut avoir dans certaines applications (trous de couple pendant les transitoires de vitesse, et par conséquent dégradation des performances de la boucle de vitesse). L'influence de la boucle de vitesse sur la boucle de couple peut en effet être telle, dans certaines applications, que l'abandon des structures de contrôle à boucles successives peut s'avérer nécessaire. Dans le cas de la machine synchrone, ne plus négliger la dynamique de la vitesse nous amène à un modèle d'état non linéaire (1).

Des études récentes ont montré l'efficacité des méthodes de commande non linéaires d'actionneurs tels que la machine à réluctance variable [11], la machine asynchrone [12] ou la machine synchrone [13]. Ces techniques (introduites dans [14]), permettent en effet d'obtenir en boucle fermée un comportement linéaire et découplé des grandeurs à asservir.

La commande non linéaire en vitesse d'une machine synchrone est malheureusement peu robuste vis-à-vis des erreurs de mesure sur la vitesse du moteur [15] (la qualité de cette mesure n'est pas toujours garantie).

Après un bref rappel du principe de la commande non linéaire en vitesse du moteur synchrone, et des problèmes de robustesse associés, nous proposons dans cet article une méthode permettant d'améliorer robustesse et performances de la commande non linéaire, mettant en jeu un calcul de trajectoire optimale à poursuivre par les grandeurs régulées (le suivi de cette trajectoire permettra une augmentation notable des gains, d'où l'amélioration de robustesse, voir $\$ 2$ ), et un estimateur du couple résistant.

Des simulations et des essais expérimentaux sont présentés afin de valider la méthode proposée.

\section{Commande non linéaire en vitesse. Robustesse.}

Le servomoteur synchrone est décrit par l'équation d'état suivante, où les variables sont référencées dans le repère $D Q$ (transformation de Park, voir [7]) :

$$
\frac{\mathrm{d} x}{\mathrm{~d} t}=\mathbf{f}(\mathbf{x})+G \mathbf{u}
$$

avec pour vecteur d'état :

$$
\mathbf{x}=\left[\begin{array}{l}
x_{1} \\
x_{2} \\
x_{3}
\end{array}\right], \quad x_{1}=i_{d}, \quad x_{2}=i_{q}, \quad x_{3}=\Omega
$$

et comme vecteur d'entrée :

$$
\mathbf{u}=\left[\begin{array}{l}
u_{1} \\
u_{2}
\end{array}\right], \quad u_{1}=v_{d}, \quad u_{2}=v_{q}
$$


L'équation d'état est non linéaire. En effet :

$$
\mathbf{f}=\left\{\begin{array}{l}
f_{1}(\mathbf{x}) \\
f_{2}(\mathbf{x}) \\
f_{3}(\mathbf{x})
\end{array}\right\}
$$

avec :

$$
\begin{aligned}
& f_{1}(\mathbf{x})=-\frac{R}{L_{d}} x_{1}+p \frac{L_{q}}{L_{d}} x_{2} x_{3} \\
& f_{2}(\mathbf{x})=-p \frac{L_{d}}{L_{q}} x_{1} x_{3}-\frac{R}{L_{q}} x_{2}-p \frac{\Phi_{\mathrm{f}}}{L_{q}} x_{3} \\
& f_{3}(\mathbf{x})=\frac{p\left(L_{d}-L_{q}\right)}{J} x_{1} x_{2}+p \frac{\Phi_{\mathrm{f}}}{J} x_{2}-\frac{f}{J} x_{3}-\frac{C_{\mathrm{r}}}{J}=\frac{C-C_{\mathrm{r}}}{J}-\frac{f}{J} x_{3}
\end{aligned}
$$

et :

$$
\boldsymbol{G}=\left[\begin{array}{ll}
\frac{1}{L_{d}} & 0 \\
0 & \frac{1}{L_{q}} \\
0 & 0
\end{array}\right]
$$

Les symboles utilisés sont répertoriés dans le paragraphe 6 .

Afin d'obtenir une linéarisation entrée-sortie totale, c'est-à-dire sans apparition d'une dynamique de zéros éventuellement instable [14], nous choisissons comme sortie (c'est-à-dire comme variables à contrôler) $i_{d}$ et la vitesse $\Omega$.

$$
\begin{aligned}
& y_{1}=i_{d} \\
& y_{2}=\Omega .
\end{aligned}
$$

L'objectif de contrôle est de maintenir à zéro le courant $i_{d}$ et d'asservir la vitesse du moteur à sa référence $\Omega_{\text {ref }}$. Le courant $i_{d}$ étant forcé à zéro, le couple moteur ne dépend plus que du courant $i_{q}$ (voir l'expression du couple (7)). Pour les brusques variations de $\Omega_{\text {ref }}$, nous verrons qu'il est judicieux d'imposer à $\Omega$ une certaine trajectoire (notée $\Omega_{\text {tr }}$ ) lui permettant de rejoindre $\Omega_{\text {ref }}$ en respectant certaines contraintes sur le courant $i_{q}$.

L'algorithme de commande non linéaire (décrit dans [13]) que nous rappelons brièvement dans cet article doit permettre la poursuite de la trajectoire-consigne $\Omega_{\text {tr }}$, calculée en temps

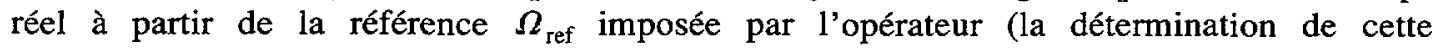
trajectoire-consigne est l'objet du paragraphe 4).

Partant de l'équation d'état (1), nous pouvons écrire :

$$
\left[\begin{array}{c}
\frac{\mathrm{d} y_{1}}{\mathrm{~d} t} \\
\frac{\mathrm{d} y_{2}^{2}}{\mathrm{~d} t^{2}}
\end{array}\right]=B_{0}+A_{0}\left[\begin{array}{c}
V_{d} \\
V_{q}
\end{array}\right]
$$


avec

$$
B_{0}=\left[\begin{array}{c}
f_{1} \\
\frac{1}{J}\left\{p f_{1} x_{2}\left(L_{d}-L_{q}\right)+p f_{2} x_{1}\left(L_{d}-L_{q}\right)+p \Phi_{\mathrm{f}} f_{2}-f f_{3}-\frac{\mathrm{d}}{\mathrm{d} t}\left(C_{\mathrm{r}}\right)\right\}
\end{array}\right]
$$

et

$$
A_{0}=\left[\begin{array}{cc}
\frac{1}{L_{d}} & 0 \\
\frac{p}{L_{d} J}\left(L_{d}-L_{q}\right) x_{2} & \frac{p}{L_{q} J}\left(\left(L_{d}-L_{q}\right) x_{1}+\Phi_{\mathrm{f}}\right)
\end{array}\right] .
$$

Nous désirons une poursuite asymptotique de la trajectoire-consigne, avec une dynamique sur l'erreur décrite par l'équation :

$$
\begin{array}{lll}
\frac{\mathrm{d} e_{1}}{\mathrm{~d} t}=K_{11} e_{\mathrm{l}} & \text { avec } & e_{1}=y_{1 \mathrm{rr}}-y_{1} \\
\frac{\mathrm{d} e_{2}^{2}}{\mathrm{~d} t^{2}}=K_{21} \frac{\mathrm{d} e_{2}}{\mathrm{~d} t}+K_{22} e_{2} & \text { avec } & e_{2}=y_{2 \mathrm{tr}}-y_{2}
\end{array}
$$

où $y_{1 \text { tr }}$ et $y_{2 \text { tr }}$ représentent les trajectoires-consignes pour les sorties $y_{1}$ et $y_{2}$ (qui sont, on le rappelle, $i_{d}$ et $\Omega$ ). Il est aisé de placer les pôles du système, en ajustant les coefficients $K_{11}, K_{21}$ et $K_{22}$.

Si le déterminant de la matrice de découplage $A_{0}$ est non nul (il suffit pour cela que $i_{d}$ n'atteigne pas le courant de désexcitation (voir annexe et [13]), nous pouvons alors appliquer la loi de commande :

$$
\left[\begin{array}{l}
V_{d} \\
V_{q}
\end{array}\right]=A_{0}^{-1}\left[-B_{0}+\left[\begin{array}{c}
K_{11}\left(y_{1 \mathrm{tr}}-y_{1}\right) \\
K_{21} \frac{\mathrm{d}}{\mathrm{d} t}\left(y_{2 t}-y_{2}\right)+K_{22}\left(y_{2 t r}-y_{2}\right)
\end{array}\right]\right]
$$

Dans l'expression (11), le terme $\frac{\mathrm{d}\left(C_{\mathrm{r}}\right)}{\mathrm{d} t}$ peut être négligé si :

$$
\left|\frac{\mathrm{d} C_{\mathrm{r}}}{\mathrm{d} t}\right| \ll\left|\frac{\left(p \Phi_{\mathrm{f}}\right)^{2} \Omega}{L_{q}}\right| \text {. }
$$

Pour une vitesse moyenne de $115 \mathrm{rd} / \mathrm{s}, \mathrm{d} C_{\mathrm{r}} / \mathrm{d} t$ doit être inférieur à $9368 \mathrm{Nm} / \mathrm{s}$. Nous ferons cette approximation par la suite, sachant que si (16) n'était pas vérifiée, il faudrait calculer la dérivée du couple résistant à partir de l'estimée de ce dernier (voir paragraphe 3).

Nous obtenons ainsi les tensions de commande nécessaires :

$$
\begin{aligned}
V_{d}= & L_{d} K_{11}\left(i_{d \mathrm{tr}}-i_{d}\right)+R i_{d}-p L_{q} \Omega i_{q} \\
V_{q}= & \frac{-\left(L_{d}-L_{q}\right) L_{q}}{\left(L_{d}-L_{q}\right) I_{d}+\Phi_{\mathrm{f}}} i_{q}\left[K_{11}\left(i_{d \mathrm{tr}}-i_{d}\right)\right]+R i_{q}+p L_{d} \Omega i_{d}-p \Phi_{\mathrm{f}} \Omega \\
& +\frac{L_{q} J}{p\left(L_{d}-L_{q}\right) I_{d}+p \Phi_{\mathrm{f}}}\left[K_{21} \frac{\mathrm{d}}{\mathrm{d} t}\left(\Omega_{\mathrm{tr}}-\Omega\right)+K_{22}\left(\Omega_{\mathrm{tr}}-\Omega\right)+\frac{f}{J} \frac{\mathrm{d} \Omega}{\mathrm{d} t}\right] .
\end{aligned}
$$


Nous trouvons sur la figure 1 le schéma général du processus. Le modèle d'état du moteur est lié aux grandeurs triphasées accessibles par une transformation de Park (pour les tensions) et une transformation inverse de Park (pour les courants). La matrice $P(p \theta)$ (transformation de Park) est définie dans le paragraphe 6. La machine est alimentée par des tensions triphasées fournies par un onduleur fonctionnant en modulation de largeur d'impulsions, et les grandeurs de sortie accessibles sont les courants triphasés, la vitesse et la position. L'algorithme de contrôle se charge :

- de reconstruire les variables d'état $i_{d}$ et $i_{q}$ à partir des courants mesurés (transformation de Park),

- de calculer les tensions $V_{d}$ et $V_{q}$ nécessaires pour la commande du processus (application de (17)),

- de faire la transformation inverse de Park de $\left[\begin{array}{l}V_{d} \\ V_{q}\end{array}\right]$, afin de calculer les tensions triphasées à imposer au moteur par le biais de la modulation de largeur d'impulsions.

Nous pouvons voir sur les figures 2 et 3 le comportement de la commande non linéaire en vitesse, lorsque nous prenons directement pour trajectoire-consigne $\Omega_{\mathrm{tr}}$, la référence $\Omega_{\text {ref }}$.

Influence des gains sur la robustesse de la commande et l'amplitude de $i_{q}$.

Nous remarquons sur la figure 2 que la robustesse de la commande non linéaire vis-à-vis de la qualité de la mesure de vitesse dépend fortement des gains $K_{21}$ et $K_{22}$.

i) pour les faibles gains $\left(K_{21}=140\right.$ et $K_{22}=10000$ ), la commande est peu robuste : erreur de $+47 \% /-24,3 \%$ sur la grandeur régulée $(\Omega)$, alors qu'il n'y a qu'une erreur de $\pm 5 \%$ sur la mesure de vitesse;

ii) pour des gains plus importants $\left(K_{21}=240\right.$ et $\left.K_{22}=40000\right)$, la robustesse est sensiblement améliorée, puisque pour la même erreur de $\pm 5 \%$ sur la mesure de vitesse, celle-ci est régulée à une valeur beaucoup plus proche de sa référence $(+4,4 \% /-4,1 \%)$.

La figure 3 montre que le choix de ces gains influera également sur l'amplitude du pic sur $i_{q}$. Or nous savons que le courant $i_{q}$ ne doit pas dépasser un certain courant $I_{q_{\max }}$ maximal admissible par le moteur (voir annexe). Les gains $K_{21}=240$ et $K_{22}=40000$ sont donc trop importants, car le pic sur $i_{q}$ dépasse $I_{q_{\max }}$

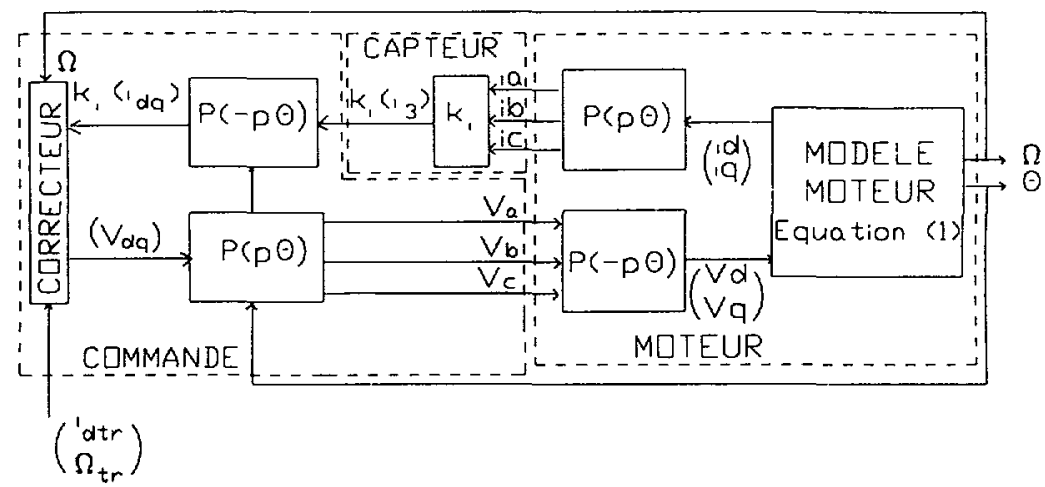

Fig. 1. - Schéma général du processus.

[General scheme of the system.] 


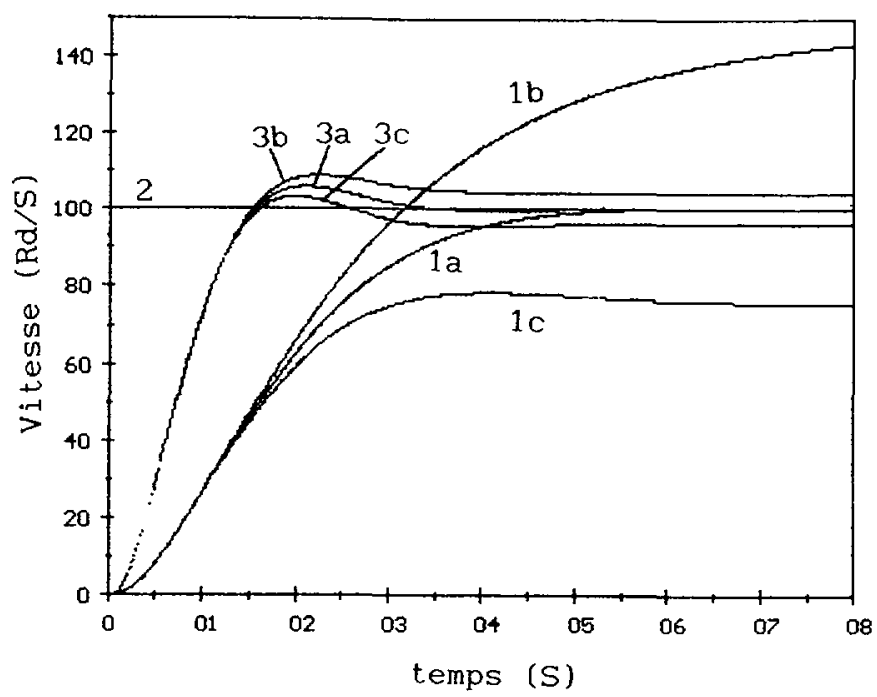

Fig. 2. - Commande non linéaire en vitesse : courbe $2: \Omega_{\text {ref }}$; courbes $1: K_{21}=140, K_{22}=10000$; courbes $3: K_{21}=240, K_{22}=40000$; courbes indexées a) pas d'erreur sur la mesure de vitesse ; courbes indexées b) erreur de $5 \%$ sur la mesure de vitesse ; courbes indexées c) erreur de $-5 \%$ sur la mesure de vitesse.

[Nonlinear speed control : curve 2: $\Omega_{\text {ref }}$, curves $1: K_{21}=140, K_{22}=10000 ;$ curves $3: K_{21}=240$, $K_{22}=40000$; curves indexed a) no error on the speed measurement; curves indexed b) $5 \%$ error on the speed measurement; curves indexed c) $-5 \%$ error on the speed measurement.]

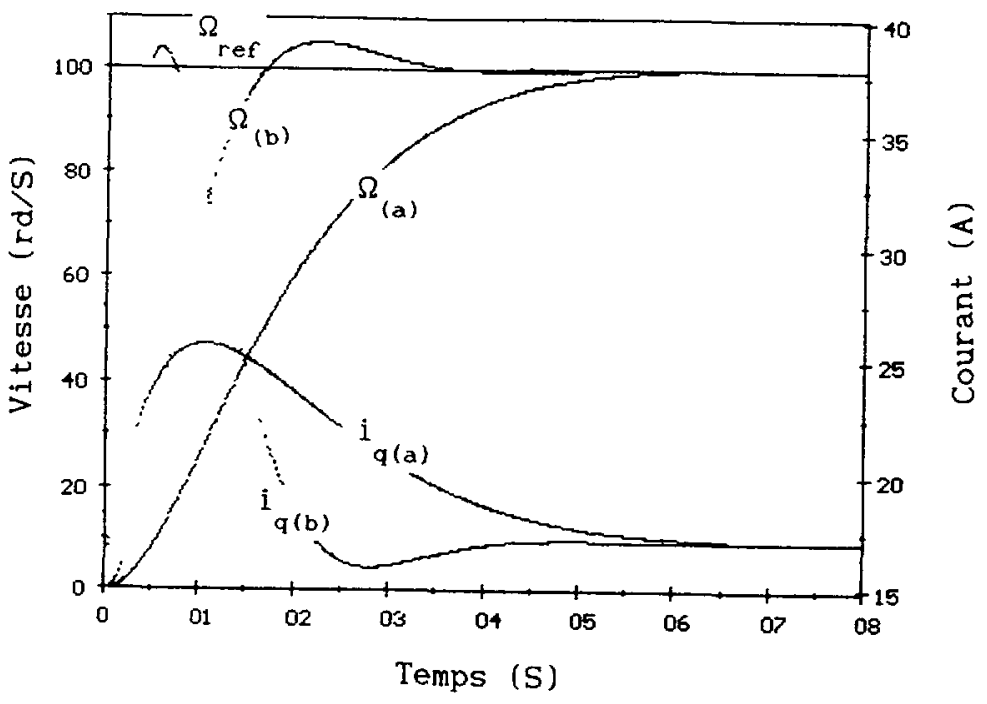

Fig. 3. - Commande non linéaire en vitesse. Influence des gains sur l'amplitude de $i_{q}$, $C_{\mathrm{r}}=8 \mathrm{Nm}$; courbes indexées a) $K_{21}=140, K_{22}=10000$; courbes indexées b) $K_{21}=240$, $K_{22}=40000$.

[Nonlinear speed control. Dependence of $i_{q}$ on the gains ; $C_{\mathrm{r}}=8 \mathrm{Nm}$; curves indexed a) $K_{21}=140, K_{22}=10000$; curves indexed b) $K_{21}=240, K_{22}=40000$.] 
La méthode proposée par la suite (commande non linéaire avec calcul de trajectoire et estimation du couple résistant), rend indépendants l'amplitude des gains de la commande et cette contrainte sur $i_{q}$. Les gains ne sont alors plus limités que par des critères de stabilité (évoqués dans [16]) et la robustesse de la commande est améliorée. Un autre objectif de cette méthode sera d'amener $i_{q}$ à $\pm I_{q_{\max }}$ (défini dans l'annexe) et de l'y maintenir pendant tout transitoire de vitesse. Le couple moteur étant ainsi optimisé, le temps de réponse à toute sollicitation de vitesse sera optimal.

\section{Estimation de l'accélération et du couple résistant.}

Dans l'équation (17), donnant la commande nécessaire à la linéarisation et au découplage de notre système intervient l'accélération $\mathrm{d} \Omega / \mathrm{d} t$, elle-même fonction du couple de charge (voir (1)). Or, ces variables ne sont pas facilement mesurables. Nous proposons donc deux méthodes pour pallier cet inconvénient :

a) la première méthode consiste à remplacer, dans l'expression des tensions de commande nécessaires, $\mathrm{d} \Omega / \mathrm{d} t$ par son estimation. Nous rappelons que le processeur de signal dédié au contrôle numérique de la machine peut estimer grossièrement cette accélération, en retranchant à la valeur courante de $\Omega_{\mathrm{m}}$, la valeur de la vitesse mesurée lors de la période d'échantillonnage précédente, puis en divisant la différence ainsi obtenue par la période d'échantillonnage ;

b) la deuxième méthode consiste à estimer $C_{\mathrm{r}}$.

La première des deux stratégies a été simulée et expérimentée, et n'a pas donné de résultats concluants, car l'estimation de l'accélération est trop grossière. En effet, pour une accélération maximale (définie dans l'annexe) de $\gamma=12730 \mathrm{rd} / \mathrm{s}^{2}$, pour une période d'échantillonnage $T_{\mathrm{e}}=400 \mu$ s (justifiée dans le $\$ 5.2$ ), nous avons, entre deux échantillons de vitesse une différence de $\Delta \Omega=5,1 \mathrm{rd} / \mathrm{s}$. Notre convertisseur analogique-numérique 8 bits donne donc une différence de 2 digits entre deux échantillons. La mesure donnée par le convertisseur présentant une erreur systématique de $\pm 0,5$ digit, nous obtenons donc une erreur de $25 \%$ sur l'estimation de l'accélération.

Le principe de la seconde méthode est présenté sur la figure 4.

Une estimation de la vitesse $\hat{\Omega}$ est faite à partir :

* d'une reconstitution du couple moteur de la machine

$$
C=\left[p\left(L_{d}-L_{q}\right) i_{d_{\mathrm{m}}}+p \Phi_{\mathrm{f}}\right] i_{q_{\mathrm{m}}}
$$

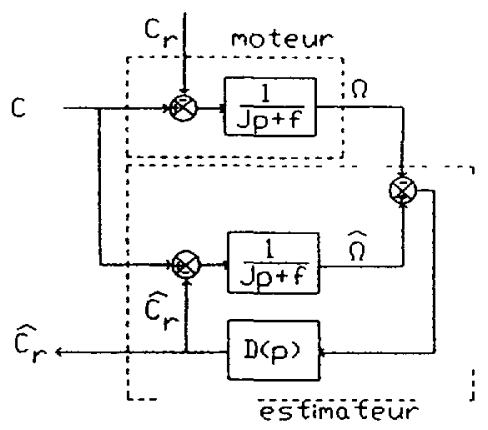

Fig. 4. - Estimateur du couple résistant.

[The load torque estimator.] 
où $i_{d_{\mathrm{m}}}$ et $i_{q_{\mathrm{m}}}$ représentent respectivement les valeurs mesurées pour les courants $i_{d}$ et $i_{q}$,

* d'une valeur estimée du couple résistant $\hat{C}_{\mathrm{r}}$.

L'erreur entre la valeur estimée de la vitesse $\hat{\Omega}$ et la vitesse mesurée $\Omega_{\mathrm{m}}$ est présentée en entrée d'un régulateur Proportionnel-Intégral numérique $D(p)$ calculant l'estimée $\hat{C}_{\mathrm{r}}$. Nous obtenons :

$$
\frac{\hat{C}_{\mathrm{r}}}{C_{\mathrm{r}}}=\frac{D(p)}{J p+f+D(p)}
$$

avec $D(p)=K_{1}+\frac{K_{2}}{p}$, on obtient :

$$
\frac{\hat{C_{\mathrm{I}}}}{C_{\mathrm{I}}}=\frac{1+\frac{K_{1}}{K_{2}} p}{1+\frac{f+K_{1}}{K_{2}} p+\frac{J}{K_{2}} p^{2}}
$$

Les pôles de la boucle d'estimation peuvent aisément être réglés. Cette deuxième stratégie donne de bons résultats (ceux-ci sont présentés dans le $\$ 5$ ).

D'autres méthodes pour estimer le couple résistant ont déjà été présentées dans [17] et [18]. La méthode présentée dans [17] considère le couple résistant comme une variable d'état à observer $[19,20]$, tandis que dans [18] une commande non linéaire adaptative, avec adaptation sur le paramètre $C_{\mathrm{r}}$, est proposée.

Cependant, ces méthodes utilisent l'hypothèse d'un couple de charge constant, ce qui n'est pas le cas de notre dispositif (accouplement élastique entre le moteur et la charge). L'estimateur que nous proposons dans cet article admet un couple résistant variable (bande passante $\sqrt{K_{2} / J}$, voir (20)). De plus, l'observateur [17] est bâti sur un modèle d'état linéaire, ce qui rend cette dernière méthode difficilement applicable sur notre système électrotechnique, non linéaire du fait de l'importance du couplage entre variables électrique et mécanique. Nous avons en effet déjà montré [10] les déficiences d'une commande linéaire sur notre système (affaissement du couple pendant les accélérations).

\section{Sensibilité de l'estimateur aux erreurs paramétriques :}

Pour estimer le couple résistant, il est nécessaire de connaître les paramètres mécaniques $J$ et $f$.

- Nous pouvons voir sur la figure 10 la conséquence d'une mauvaise connaissance de $J$ sur l'estimation du couple résistant ainsi que sur le comportement de l'asservissement de vitesse. La trajectoire-consigne est ici calculée suivant le principe présenté dans le paragraphe 4. Nous constatons que :

- l'estimateur ne présente une erreur que pendant les transitoires de vitesse (cette erreur restant faible);

- le couple moteur obtenu pendant le transitoire de vitesse est trop important lorsque $J$ est sous-évalué, car la pente calculée pour la trajectoire-consigne est trop grande (voir $\S 4$ ).

Sur la figure 11 , on constate que l'influence d'une mauvaise connaissance de $f$ reste faible sur l'estimation du couple résistant. 
On voit donc, d'après ces deux figures l'intérêt d'une connaissance précise de $J$ (calcul ou mesure préalable). Pour les systèmes à inertie variable, il peut être intéressant d'avoir recours à une commande adaptative, avec adaptation sur le paramètre $J$ [21].

\section{Détermination de la trajectoire optimale pour la vitesse.}

Nous devons donc choisir, pour toute sollicitation sur la référence de vitesse $\Omega_{\text {ref, la }}$ trajectoire-consigne optimale $\Omega_{\text {tr }}$, vers laquelle la vitesse $\Omega$ devra converger asymptotiquement. Nous choisirons une trajectoire-consigne permettant à la vitesse d'atteindre le plus rapidement possible sa référence, sans dépasser le courant maximal admissible par le moteur.

Si la commande non linéaire (étudiée dans le paragraphe précédent) permet aux sorties $y_{1}$ et $y_{2}$ (c'est-à-dire $i_{d}$ et $\Omega$ ) de suivre rapidement leurs trajectoires-consignes respectives $y_{1 \mathrm{tr}}=i_{d \mathrm{tr}}=I_{d \mathrm{ref}}=0$ et $y_{2 \mathrm{tr}}=\Omega_{\mathrm{tr}}$, nous obtenons, à partir de l'expression d'état (1):

$$
p \Phi_{\mathrm{f}} i_{q}=J \frac{\mathrm{d} \Omega}{\mathrm{d} t}+f \Omega+C_{\mathrm{r}}=J \frac{\mathrm{d} \Omega_{\mathrm{tr}}}{\mathrm{d} t}+f \Omega_{\mathrm{tr}}+C_{\mathrm{r}}
$$

Nous cherchons à ce que la trajectoire choisie pour $\Omega$ soit telle que le courant $i_{q}$ ne dépasse pas les contraintes maximales tolérées par le moteur:

$$
\left|i_{q}\right|<I_{q_{\max }} \quad\left(I_{q_{\max }}\right. \text { fourni dans l'annexe). }
$$

Or cette relation est vérifiée si :

$$
\left|f \frac{\mathrm{d} \Omega_{\mathrm{tr}}}{\mathrm{d} t}+f \Omega_{\mathrm{tr}}+C_{\mathrm{r}}\right|<p \Phi_{\mathrm{f}} I_{q_{\max }}
$$

Or, nous savons que :

$$
\left|J \frac{\mathrm{d} \Omega_{\mathrm{rr}}}{\mathrm{d} t}+f \Omega_{\mathrm{tr}}+C_{\mathrm{r}}\right|<\left|J \frac{\mathrm{d} \Omega_{\mathrm{tr}}}{\mathrm{d} t}+C_{\mathrm{r}}\right|+\left|f \Omega_{\mathrm{tr}}\right|<\left|J \frac{\mathrm{d} \Omega_{\mathrm{tr}}}{\mathrm{d} t}+C_{\mathrm{r}}\right|+f \Omega_{\max }
$$

en notant $\Omega_{\max }$ la vitesse maximum que le moteur puisse atteindre. Ainsi :

$$
\left|J \frac{\mathrm{d} \Omega_{\mathrm{tr}}}{\mathrm{d} t}+f \Omega_{\mathrm{tr}}+C_{\mathrm{r}}\right|<\left|J \frac{\mathrm{d} \Omega_{\mathrm{tr}}}{\mathrm{d} t}+C_{\mathrm{r}}\right|+f \Omega_{\max } .
$$

Nous déduisons de (23) et (25), que pour que $i_{q}$ ne dépasse pas le courant maximum admissible $i_{q_{\max }}$ il suffit que :

$$
\left|J \frac{\mathrm{d} \Omega_{\mathrm{tr}}}{\mathrm{d} t}+C_{\mathrm{r}}\right|+f \Omega_{\max }<p \Phi_{\mathrm{f}} I_{q_{\max }}
$$

Il suffit donc que :

$$
\frac{-p \Phi_{\mathrm{f}} I_{q_{\max }}+f \Omega_{\max }-C_{\mathrm{r}}}{J}<\frac{\mathrm{d} \Omega_{\mathrm{tr}}}{\mathrm{d} t}<\frac{p \Phi_{\mathrm{f}} I_{q_{\max }}-f \Omega_{\max }-C_{\mathrm{r}}}{J}
$$

Nous ne connaissons pas $C_{\mathrm{r}}$, mais nous disposons de son estimée (voir paragraphe précédent).

D'autre part, il est intéressant d'avoir $\left|\mathrm{d} \Omega_{\mathrm{tr}} / \mathrm{d} t\right|$ aussi grand que possible, afin que la vitesse atteigne le plus rapidement possible sa référence. 


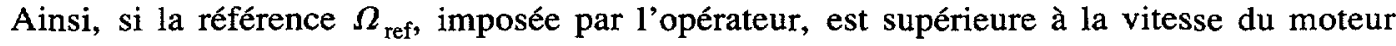
$\Omega$, nous imposerons :

$$
\frac{\mathrm{d} \Omega_{\mathrm{tr}}}{\mathrm{d} t}=\frac{p \Phi_{\mathrm{f}} I_{q_{\max }}-f \Omega_{\max }-\hat{C}_{\mathrm{r}}}{J}=\Gamma_{1 \mathrm{tr}}
$$

et donc la trajectoire-consigne :

$$
\Omega_{\mathrm{tr}}(t)=\Omega\left(t_{0}\right)+\int_{t_{0}}^{t} \Gamma_{1 \mathrm{tr}}(t) \mathrm{d} t
$$

$t_{0}$ étant l'instant où l'opérateur a changé la référence $\Omega_{\text {ref. }}$.

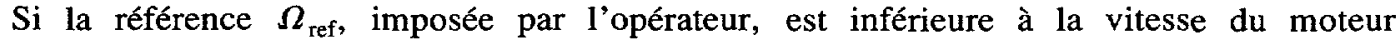
$\boldsymbol{\Omega}$, nous imposerons :

$$
\frac{\mathrm{d} \Omega_{\mathrm{tr}}}{\mathrm{d} t}=\frac{-p \Phi_{\mathrm{f}} I_{g_{\max }}+f \Omega_{\max }-\hat{C}_{\mathrm{r}}}{J}=\Gamma_{2 \mathrm{rr}}
$$

et donc la trajectoire-consigne :

$$
\Omega_{\mathrm{tr}}(t)=\Omega\left(t_{0}\right)+\int_{t_{0}}^{t} \Gamma_{2 \mathrm{tr}}(t) \mathrm{d} t
$$

Ainsi la vitesse du moteur, en suivant cette trajectoire-consigne, évoluera de la manière la

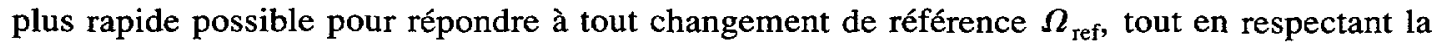
contrainte (22) sur $i_{q}$.

En conclusion, la trajectoire-consigne sera calculée en temps réel par le processeur de signal du système numérique de la façon suivante :

* si $\Omega=\Omega_{\text {ref }}$ alors $\Omega_{\text {tr }}=\Omega_{\text {ref }}$

* si $\Omega<\Omega_{\text {ref }}$ alors $\Omega_{\mathrm{tr}}=\Omega\left(t_{0}\right)+\int_{t_{0}}^{t} \Gamma_{\mathrm{Itr}}(t) \mathrm{d} t$

* si $\Omega>\Omega_{\mathrm{ref}}$ alors $\Omega_{\mathrm{tr}}=\Omega\left(t_{0}\right)+\int_{t_{0}}^{t} \Gamma_{2 \mathrm{rr}}(t) \mathrm{d} t$.

Nous pouvons voir que pour toute sollicitation de vitesse, le moteur répondra avec l'accélération maximale acceptable (d'un point de vue électrique, puisque l'accélération est liée avec $i_{q}$ ), et donc avec un couple et un courant $i_{q}$ maximaux.

Il est à noter que le temps de réponse de l'asservissement de vitesse dépend de l'amplitude de la sollicitation en vitesse. Par exemple, pour un couple $C_{\mathrm{r}}$ constant, nous avons :

$$
t_{\text {réponse }}=\frac{\Omega_{\text {ref }}-\Omega_{\text {ref précédent }}}{\Gamma_{\mathrm{tr}}}
$$

avec $\Gamma_{\mathrm{tr}}=\Gamma_{\mathrm{tr} 1}$ si $\Omega_{\text {ref }}>\Omega$, et $\Gamma_{\mathrm{tr}}=\Gamma_{\mathrm{tr} 2}$ dans l'autre cas.

Nous pouvons voir sur la figure 5 le schéma bloc représentant la structure de la commande non linéaire avec calcul de trajectoire. 


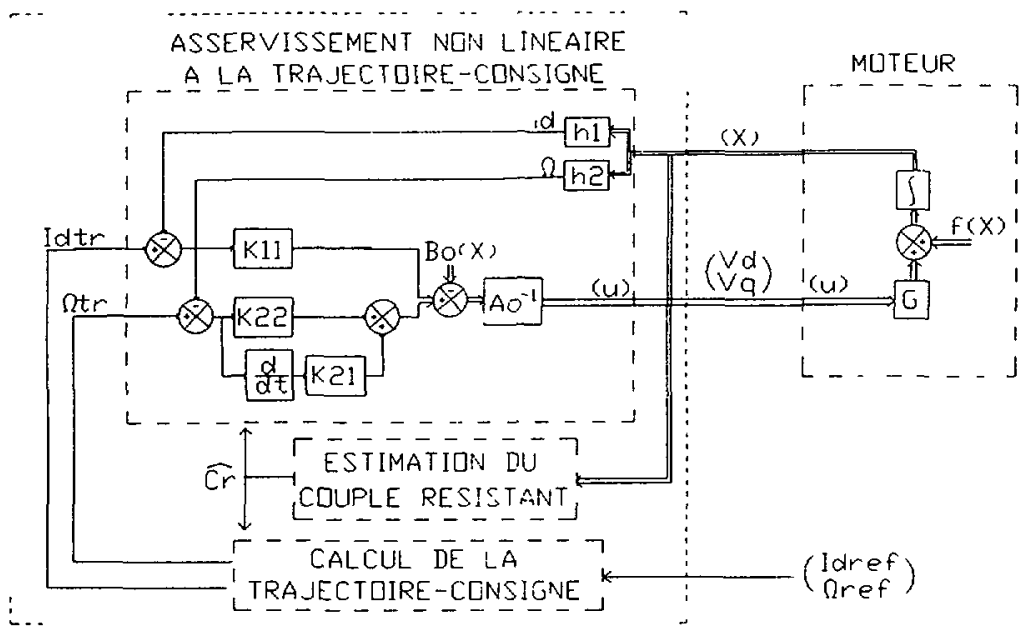

Fig. 5. - Schéma de la commande non linéaire en vitesse avec calcul de la trajectoire de vitesse et estimation du couple résistant.

[Scheme of the speed nonlinear control of a synchronous servomotor with computation of the asymptotic trajectory and estimation of the load torque.]

Augmentation de la robustesse:

Ayant contraint le courant $i_{q}$ à se maintenir à la valeur $I_{q_{\max }}$ pendant tout transitoire (par le biais du choix d'une trajectoire-consigne appropriée pour la vitesse), la valeur des gains $K_{11}, K_{21}$ et $K_{22}$ n'a plus d'effet sur l'amplitude de $i_{q}$. Nous pouvons donc augmenter ceux-ci, ce qui a pour effet une augmentation de la robustesse de la commande vis-à-vis de la mesure de vitesse (voir $\& 2$ ).

\section{Résultats.}

Nous avons utilisé pour expérimenter cette commande :

* un processeur de signal TEXAS-INSTRUMENT TMS32010 utilisé en coprocesseur arithmétique du microprocesseur INTEL 8086. La période d'échantillonnage a ainsi pu être ramenée à $400 \mu \mathrm{s}$, malgré la lourdeur du calcul nécessaire à l'algorithme ;

* un onduleur à transistors MOS, de façon à avoir des temps morts de commutation très réduits $(300 \mathrm{~ns})$. Cet onduleur fonctionne en modulation de largeur d'impulsions.

L'organe de calcul de la carte de contrôle fournit successivement, à chaque période d'échantillonnage :

* les transformations de Park des courants triphasés ;

* la trajectoire-consigne de la vitesse $\Omega_{\text {tr }}$ calculée à partir de la référence $\Omega_{\text {ref }}$ donnée par l'opérateur ;

* le résultat de l'algorithme de contrôle (c'est-à-dire les tensions $v_{d}$ et $v_{q}$ nécessaires) ;

* les rapports cycliques triphasés à imposer à l'onduleur, ceux-ci étant calculés comme étant le rapport entre les transformées inverses de Park de $V_{d}$ et $V_{q}$ nécessaires (tensions triphasées), et la tension continue de l'onduleur. 
5.1 RÉSUltats dE SIMULATION. - Les résultats de simulation présentés tiennent compte de la discrétisation des signaux, en simulant le blocage des tensions de commande entre deux périodes d'échantillonnage, et le retard pur dû au temps de calcul de l'algorithme de contrôle.

Nous avons montré dans le paragraphe 2 et grâce à la simulation figure 2, que l'influence d'une mauvaise mesure de vitesse est notablement diminuée par l'emploi de gains $K_{11}, K_{21}$ et $K_{22}$ importants, ce qui n'est rendu possible que par le calcul en temps réel de la trajectoire asymptotique pour la vitesse. De plus nous avons montré [22], la prépondérance de la mesure de vitesse vis-à-vis des autres mesures (pour les capteurs de courant, de vitesse et de position utilisés sur notre banc d'essais) sur la robustesse de la commande. C'est pourquoi, afin de ne pas surcharger les figures 6 à 11, les capteurs sont supposés parfaits lors des simulations correspondantes.

Nous présentons, sur les figures $6,7,8$ et 9 la simulation d'un essai indiciel sur la vitesse du moteur, $\Omega_{\text {ref }}$ passant de $-120 \mathrm{rd} / \mathrm{s}$ à $120 \mathrm{rd} / \mathrm{s}$, pendant que $i_{d \text { ref }}$ reste à $0 \mathrm{~A}$.

La simulation a été faite avec $K_{11}=800, K_{21}=240$, et $K_{22}=40000$. La figure 6 est relative à un échelon sur $\Omega_{\text {ref }}$ pour un couple résistant nul, tandis que pour les figures 7,8 et 9 , le couple résistant est constant et vaut $8 \mathrm{Nm}$. On remarque qu'en régime permanent $i_{q}$ se stabilise à $\frac{C_{\mathrm{r}}}{p \Phi_{\mathrm{f}}}$.

La figure 8 montre l'évolution de $\hat{\Omega}$, tandis que la figure 9 montre celle de $\hat{C}_{\mathrm{r}}$ pendant le transitoire de vitesse : on constate sur la figure 9 que l'erreur d'estimation sur le couple résistant est faible.

On constate également que le courant maximal $i_{q}$ atteint pendant le transitoire diffère peu, que le moteur soit chargé ou à vide (comparer les Figs. 6 et 7). On constate néanmoins un léger dépassement par rapport au créneau de courant prévu, ce dépassement étant plus important

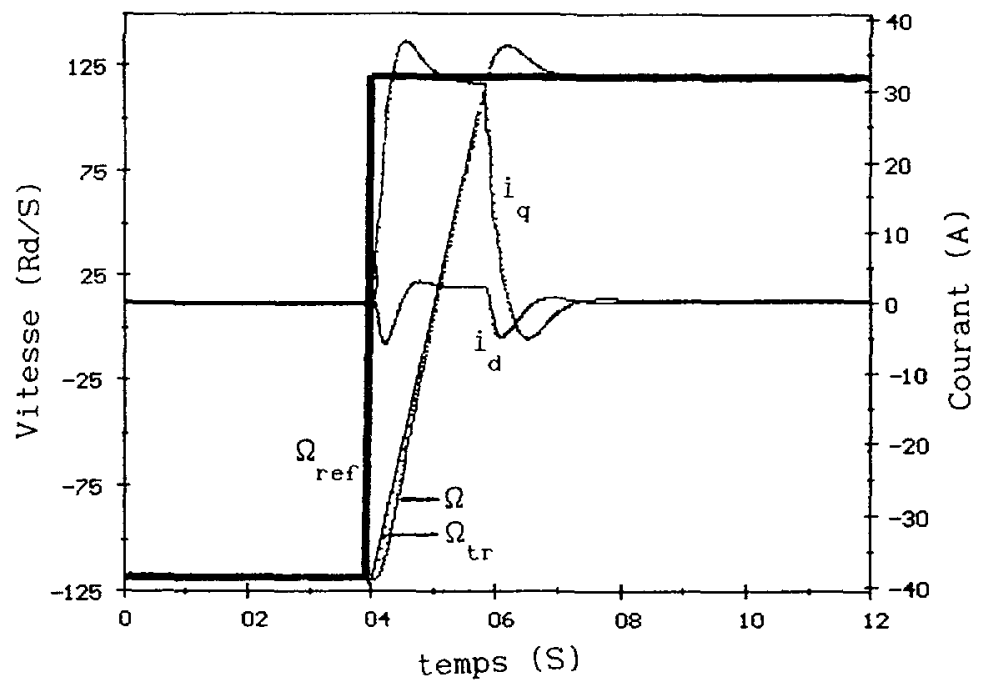

Fig. 6. - Commande non linéaire avec calcul de trajectoire et estimation du couple résistant $C_{\mathrm{r}}=0 \mathrm{Nm}$.

[Simulation of the speed nonlinear control of a synchronous servomotor with computation of the asymptotic trajectory and estimation of the load torque, $C_{\mathrm{r}}=0 \mathrm{Nm}$.] 


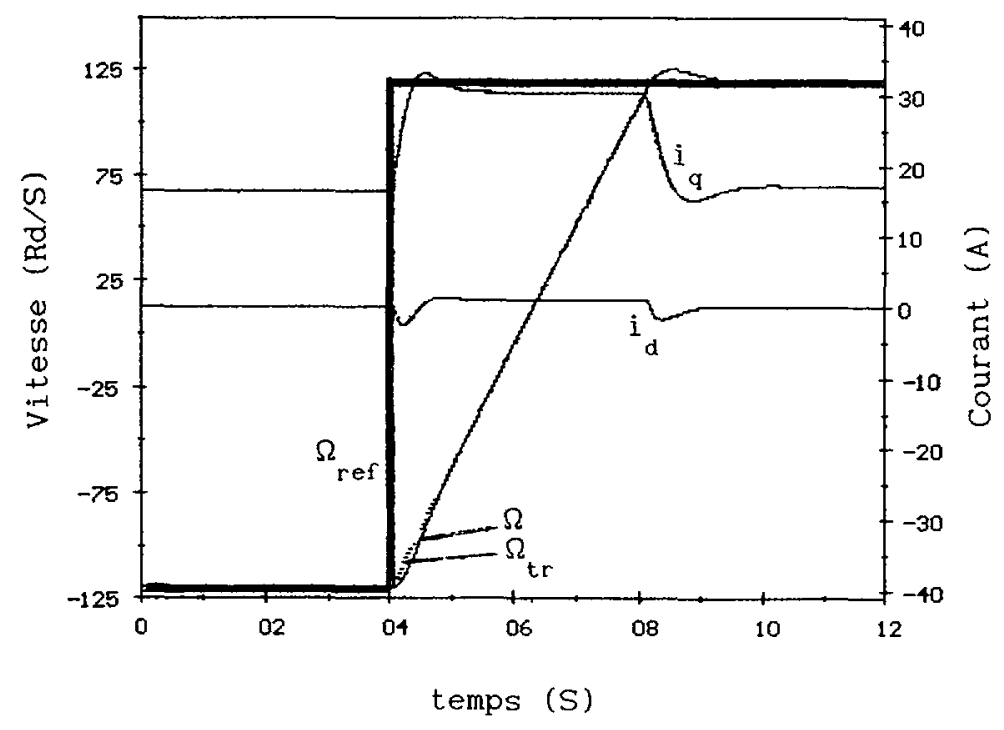

Fig. 7. - Commande non linéaire avec calcul de trajectoire et estimation du couple résistant $C_{\mathrm{r}}=8 \mathrm{Nm}$.

[Simulation of the speed nonlinear control of a synchronous servomotor with computation of the asymptotic trajectory and estimation of the load torque, $C_{\mathrm{r}}=8 \mathrm{Nm}$.]

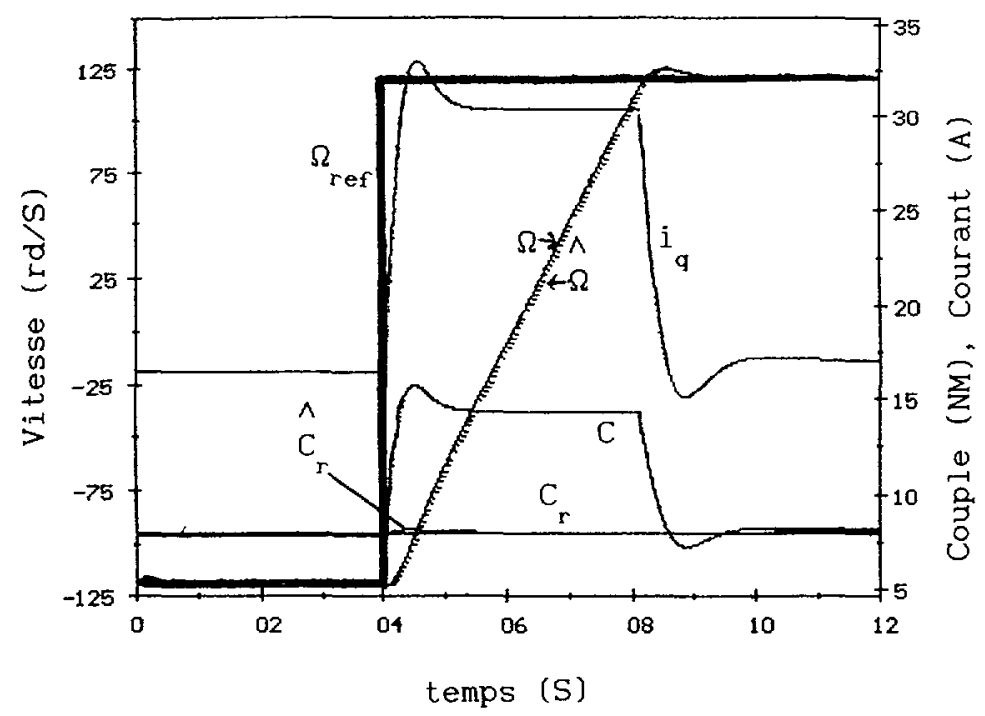

Fig. 8. - Comportement de l'estimateur de couple résistant pendant les transitoires de vitesse $C, \Omega$ et $\hat{\Omega}$.

[Behaviour of the load torque estimator during the speed transients $C, \boldsymbol{\Omega}$ and $\hat{\boldsymbol{\Omega}}$.] 


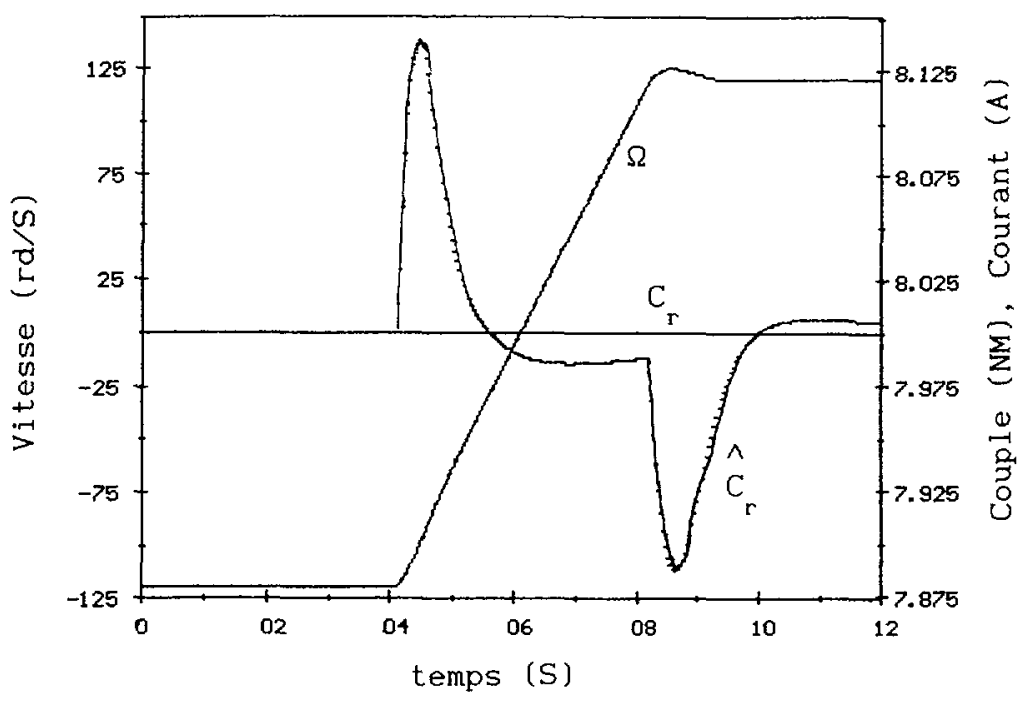

Fig. 9. - Comportement de l'estimateur de couple résistant pendant les transitoires de vitesse $\Omega, C_{\text {r }}$ et $\hat{C}_{\text {r }}$

[Behaviour of the load torque estimator during the speed transients $\Omega, C_{\mathrm{r}}$ and $\hat{C}_{\mathrm{r}}$.]

dans le cas où le couple résistant est nul (ce dépassement pourrait être sensiblement diminué par l'emploi d'une trajectoire de vitesse où la rupture de pente serait adoucie (spline), et par une commande discrète du type multirate [23]). Quel que soit le couple résistant, le temps de réponse à $5 \%$ de la vitesse mécanique $\Omega$ est optimal $\left(20 \mathrm{~ms}\right.$ pour $C_{\mathrm{r}}=0 \mathrm{Nm}, 45 \mathrm{~ms}$ pour $C_{\mathrm{r}}=8 \mathrm{Nm}$ ). Il ne pourrait être plus rapide sans augmenter le courant $i_{q}$, ce qui n'est pas acceptable pour le moteur.

5.2 RÉSUltatS EXPÉRIMENTAUX. - La mise en œuvre d'une telle commande non linéaire nécessite un volume de calcul important, et par voie de conséquence l'emploi d'un calculateur du type processeur de signal s'impose, pour réduire de façon importante le temps de calcul, et travailler ainsi avec une période d'échantillonnage acceptable. La période d'échantillonnage est pour cette expérimentation de $400 \mu \mathrm{s}$, et la période de hachage de l'onduleur (ce dernier travaille en modulation de largeur d'impulsions) est de $100 \mu s$. Un DSP (TEXAS-INSTRUMENT TMS32010) a été utilisé (ce processeur de signal est d'un coût relativement faible pour des performances suffisantes pour cette application (temps de calcul et de transfert de données inférieur à $300 \mu \mathrm{s}$ )).

La période d'échantillonnage $T_{\mathrm{e}}$ doit être supérieure au temps de calcul et de transfert de données : nous avons pris $T_{\mathrm{e}}=400 \mu \mathrm{s}$. La période d'échantillonnage ne pourrait être plus grande, car celle-ci doit rester faible devant:

- la constante de temps électrique dans l'axe $D: L_{d} / R=2,33 \mathrm{~ms}$,

- la constante de temps électrique dans l'axe $Q: L_{q} / R=4,67 \mathrm{~ms}$,

- la constante de temps électromécanique : $R J /\left(p \Phi_{\mathrm{f}}\right)^{2}=2,89 \mathrm{~ms}$,

- la période électrique minimale (à vitesse constante $\Omega_{\max }$ ): $2 \Pi / p \Omega_{\max }=5,36 \mathrm{~ms}$ (13 échantillons par période). 


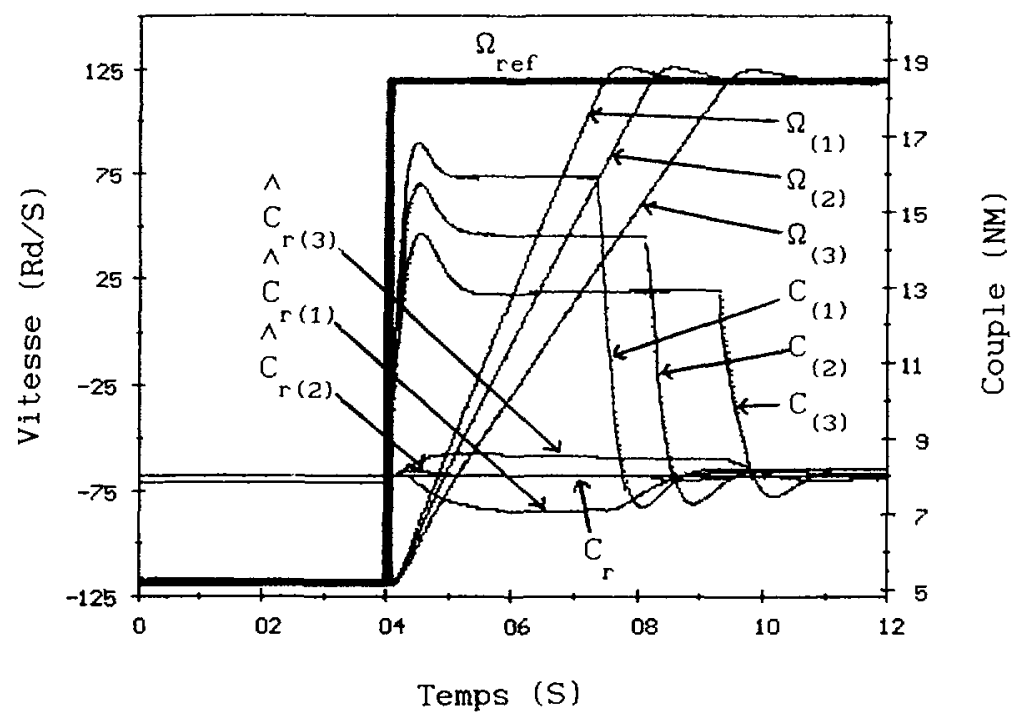

Fig. 10. - Sensibilité de l'estimateur de couple résistant à une mauvaise connaissance de $J$; courbes indexées (1): $J_{\text {algorthme }}=0,9 J$; courbes indexées (2): $J_{\text {algorthme }}=J$; courbes indexées (3): $J_{\text {algorthme }}=1,1 J$.

[Sensibility of the load torque estimator bad knowing of $J$ (inertia) ; curves indexed (1) $J_{\text {algornhm }}=0.9 J$; curves indexed (2) $J_{\text {algonthm }}=J$; curves indexed (3) $J_{\text {algorthm }}=1.1 J$.]

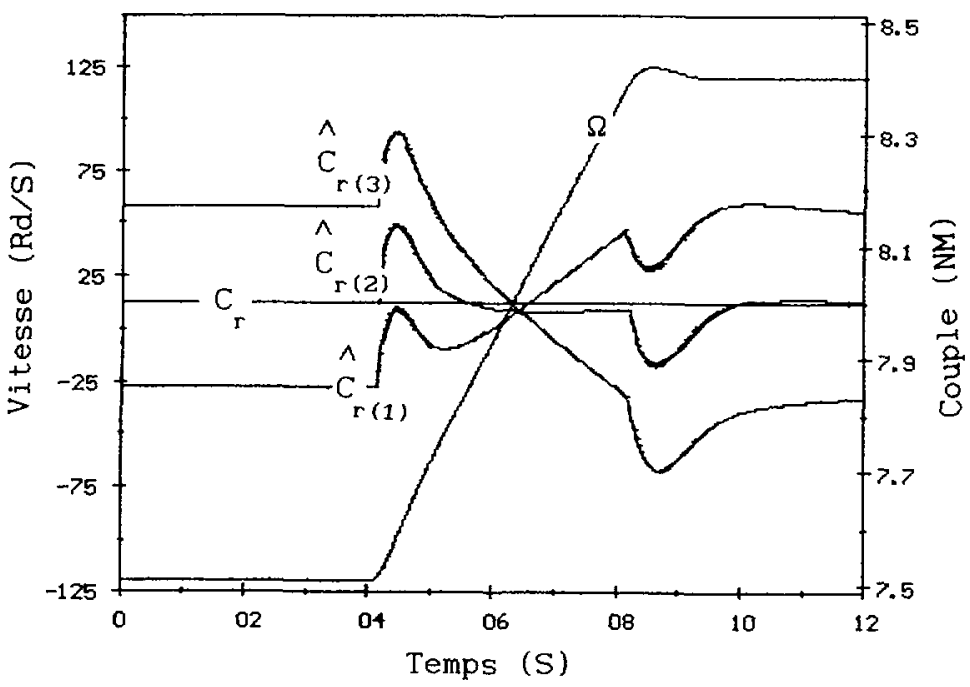

Fig. 11. - Sensibilité de l'estimateur de couple résistant, transitoire de vitesse, mauvaise connaissance de $f$ (frottement visqueux); courbes indexées (1) $f_{\text {algonthme }}=0,2 f$; courbes indexées (2) $f_{\text {algonthme }}=f$; courbes indexées (3) $f_{\text {algonthme }}=2 f$.

[Sensibility of the load torque estimator during speed transients, bad knowing of $f$ (viscous friction coefficient) ; curves indexed (1) $f_{\text {algorthm }}=0.2 f$; curves indexed (2) $f_{\text {algorthm }}=f$; curves indexed (3) $f_{\text {algonthm }}=2 f$.] 
Courants, vitesse et position sont échantillonnés à la même fréquence. En effet :

- nous ne négligeons pas la dynamique de la vitesse vis-à-vis de celle des courants (comparer les constantes de temps électriques à la constante de temps électromécanique) ;

- pour la vitesse maximale de rotation, $p \theta$ varie de 0,47 rd entre 2 échantillons $(7,5 \%$ de la période électrique). On ne peut donc accepter un échantillonnage plus lent de la position sans altérer, à haute vitesse, la validité de nos transformées de Park.

Nous pouvons voir sur les figures 12,13 et 14 un essai expérimental où $\Omega_{\text {ref }}$ passe de - $120 \mathrm{rd} / \mathrm{s}$ à $120 \mathrm{rd} / \mathrm{s}$, pour un moteur non chargé. $\Omega$ est comparé à $\Omega_{\text {tr }}$ sur la figure 12 , est superposé à $i_{d}$ sur la figure 13 et à $i_{q}$ sur la figure 14 .

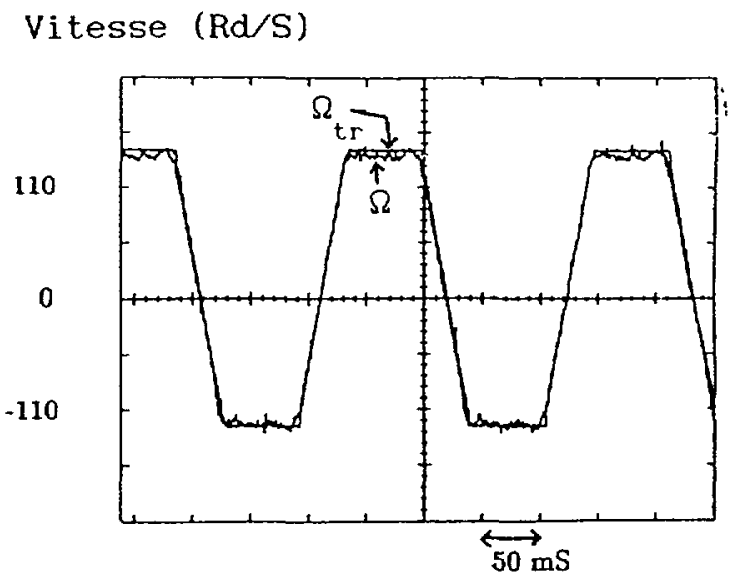

Fig. 12. - Expérimentation; essai indiciel sur la vitesse. Mesure de $\Omega_{\mathrm{tr}}$ (trajectoire-consigne) et $\Omega$.

[Experimental implementation; step experiment on the speed $\Omega_{\mathrm{tr}}$ (asymptotic trajectory) and $\Omega$.

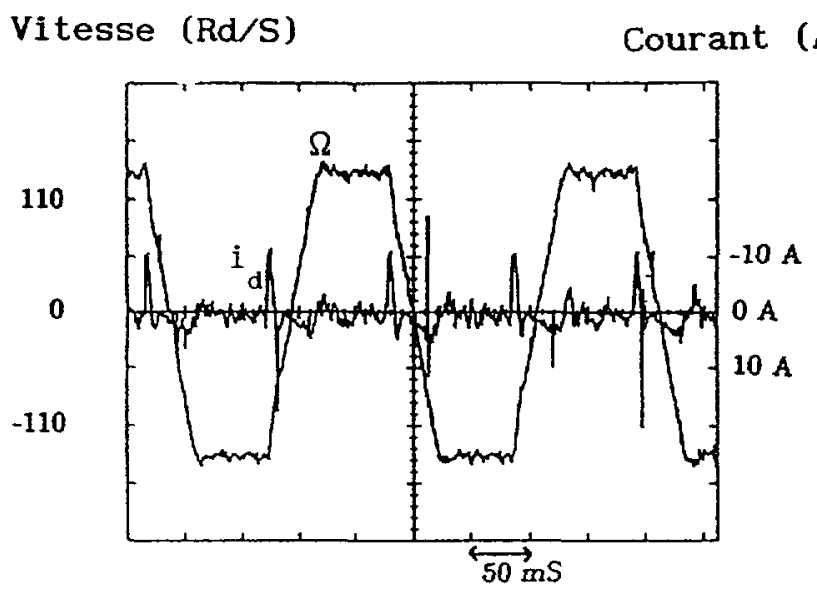

Fig. 13. - Expérimentation; essai indiciel sur la vitesse $\Omega(t)$ et $i_{d}(t)$.

[Experimental implementation; step experiment on the speed $\Omega(t)$ and $i_{d}(t)$.] 


\section{Vitesse (Rd/S) Courant (A)}

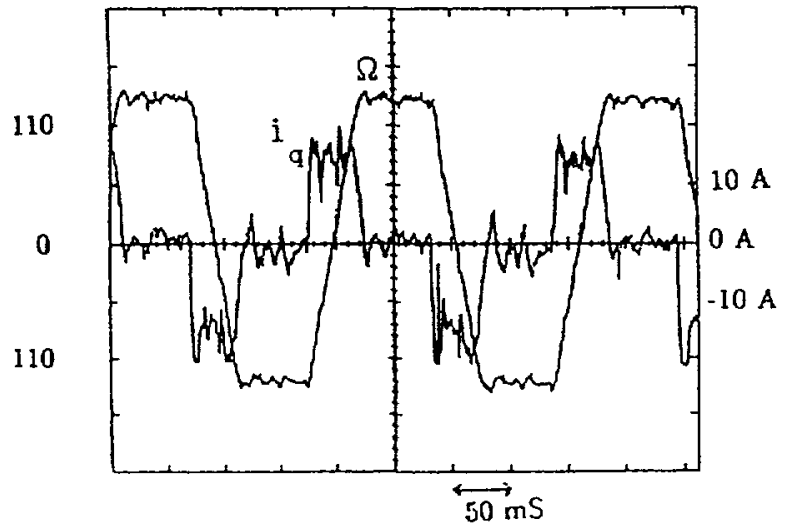

Fig. 14. - Expérimentation; essai indiciel sur la vitesse $\Omega(t)$ et $i_{q}(t)$.

[Experimental implementation; step experiment on the speed $\Omega(t)$ and $i_{q}(t)$.]

Les accélérations $\Gamma_{1 \text { tr }}$ et $\Gamma_{2 \text { tr }}$ sont calculées en temps réel en limitant $I_{q \text { max }}$ à 15 A. Afin d'éviter toute erreur statique due à une mauvaise connaissance des paramètres de la machine, des intégrateurs ont été insérés au sein des boucles de contrôle [15].

Nous pouvons donc voir sur ces résultats expérimentaux, que le temps de réponse de la vitesse mécanique est d'environ $50 \mathrm{~ms}$, que la vitesse évolue bien en suivant la trajectoireconsigne calculée en temps réel, et que $i_{q}$ monte bien au $i_{q \max }(15 \mathrm{~A})$ prédéterminé et y reste pendant toute la durée du transitoire de vitesse.

\section{Liste des symboles.}

$V_{d}$ et $V_{q}$ représentent respectivement les tensions statoriques de la machine ramenées dans les axes $D$ et $Q$ (direct et en quadrature).

$i_{d}$ est le courant, $L_{d}$ est l'inductance, $\Phi_{d}$ est le flux dans l'axe $D$.

$i_{q}$ est le courant, $L_{q}$ est l'inductance, $\Phi_{q}$ est le flux dans l'axe $Q$.

$R$ représente la résistance statorique.

$\Omega$ est la vitesse du moteur.

$p$ son nombre de paires de pôles.

$\Phi_{\mathrm{f}}$ est le flux généré par l'aimant rotorique.

$J$ est l'inertie du système.

$f$ le coefficient de frottement visqueux du système.

$C_{\mathrm{r}}$ le couple résistant.

$C$ le couple moteur.

$$
P(\xi)=\sqrt{2 / 3}\left[\begin{array}{cc}
1 & 0 \\
-1 / 2 & \sqrt{3 / 2} \\
-1 / 2 & -\sqrt{3 / 2}
\end{array}\right]\left[\begin{array}{lr}
\cos (\xi) & -\sin (\xi) \\
\sin (\xi) & \cos (\xi)
\end{array}\right] \text { est la transformation de Park }
$$




\section{Conclusion.}

Nous avons présenté dans cet article différentes variantes de commandes non linéaires basées sur une représentation d'état d'un système composé d'une machine synchrone à aimants permanents, associée à un onduleur triphasé. Ces commandes non linéaires prennent en compte la dynamique de la vitesse $\Omega$, et permettent donc au variateur d'éviter les trous de couple dus à cette dynamique (voir [10] pour plus de détails sur ces trous de couple).

Ces commandes nécessitent néanmoins l'utilisation d'un organe de calcul rapide, afin de prendre en charge l'algorithme de régulation, tout en n'augmentant pas la période d'échantillonnage de manière trop importante.

La première de ces commandes $(\S 2)$ permet d'asservir $i_{d}$ à zéro et de suivre la consigne $\Omega_{\text {ref }}$ avec un comportement du second ordre prédéterminé. Nous avons vu que le temps de réponse de cette commande (lié aux gains de l'algorithme) est limité par le courant maximal accepté par le moteur. Nous avons également constaté que ce correcteur n'est pas robuste aux erreurs de mesure sur la vitesse $\Omega$.

Nous avons ensuite présenté l'étude et l'expérimentation d'une variante de commande non linéaire, intégrant le calcul en temps réel d'une trajectoire à poursuivre $\Omega_{\text {tr }}$ à partir de la référence $\Omega_{\text {ref }}$ fournie par l'opérateur. Les entrées de notre système, linéarisé et découplé par la commande non linéaire, ne sont pas directement les références $i_{d \text { ref }}$ et $\Omega_{\text {ref, mais des }}$ trajectoires-consignes $\Omega_{\square}$ et $i_{d \text { tr }}$ calculées en temps réel par le processeur de signal utilisé pour cette commande numérique.

La principale caractéristique de cette régulation est que la vitesse mécanique $\Omega$ évolue toujours avec l'accélération maximale acceptable pour le moteur, pour toute sollicitation sur $\Omega_{\text {ref. }}$ Par conséquent, le temps de réponse de l'asservissement de vitesse est optimal, quelle que soit l'amplitude de l'essai indiciel et quel que soit le couple résistant. De plus, il n'y a pas de pertes de contrôle possible des grandeurs asservies, pertes de contrôle que nous rencontrions avec les correcteurs traditionnels pour les grandes variations de références (pour les grands mouvements les commandes traditionnelles entrent en saturation, et notre système est alors en boucle ouverte).

Cette commande non linéaire impose une contrainte sur les courants du moteur ( $i_{d}$ asservi à zéro; $i_{q}$ porté à $I_{q \max },-I_{q \max }$ ou $C_{\mathrm{r}} / p \Phi_{\mathrm{f}}$ ). Les gains de cet asservissement (et par voie de conséquence sa robustesse) peuvent être fortement augmentés, en comparaison à ceux utilisés dans les commandes non linéaires usuelles, où ils sont limités par l'amplitude maximale du pic sur $i_{q}$.

Les bons résultats expérimentaux montrent que cet algorithme peut être implanté aisément (si l'on dispose d'un organe de calcul rapide), et valident des résultats de simulation très prometteurs.

\section{Annexe.}

\section{CARACTÉRISTIQUES DU MOTEUR :}

MOTEUR SYNCHRONE AUXILEC T8-98-55/EAU.

Courant transitoire maximal $I_{q_{\max }}=30$ A.

Couple maximal transitoire $=14 \mathrm{Nm}$. Couple permanent maximum à l'arrêt $=10 \mathrm{Nm}$.

Couple maximum à $2200 \mathrm{tr} / \mathrm{min}=C_{\mathrm{NOM}}=8,5 \mathrm{Nm}$.

$R_{\mathrm{s}}=0,6 \Omega, L_{d}=1,4 \mathrm{e}^{-3} \mathrm{H} . L_{q}=2,8 \mathrm{e}^{-3} \mathrm{H}, p=4, \Phi_{\mathrm{f}}=0,12 \mathrm{WB}$.

Inertie $J_{\mathrm{m}}=11 \times 10^{-4} \mathrm{~kg} \mathrm{~m}^{2}$.

Vitesse à vide $\Omega_{\mathrm{MAX}}=293 \mathrm{rd} / \mathrm{s}$.

Vitesse nominale $\Omega_{\mathrm{NOM}}=230 \mathrm{rd} / \mathrm{s}$.

Frottement visqueux : $f=1,4 \times 10^{-3} \mathrm{Nms}$. 
A partir de ces valeurs nous pouvons calculer la valeur du courant de désexcitation : $I_{d \text { Désexcitation }}=\frac{\Phi_{\mathrm{f}}}{L_{q}-L_{d}}=85 \mathrm{~A}$ (voir [13]).

Valeur maximale acceptable pour l'accélération (pour un moteur non chargé) : $[\mathrm{d} \Omega / \mathrm{d} t]_{\max }=C_{\max } / J=12728 \mathrm{rd} / \mathrm{s}$.

CAPTEURS UTILISÉs :

- courants : sondes à effet Hall associées à des convertisseurs analogique numérique 8 bits,

- vitesse : génératrice tachymétrique associée à un convertisseur analogique numérique 8 bits,

- position : codeur incrémental 1024 points/tour.

\section{Remerciements.}

Nous remercions D. Normand-Cyrot, J.-P. Louis et S. Monaco pour l'aide apportée à l'accomplissement de ce travail.

\section{Bibliographie}

[1] Oumamar A., Louis J. P. et El-Hefnawy A., « Design of an optimal, autoadaptative current loop for D-C motor. Realisation with an hybrid device including a microprocessor $\gg, 2$ nd I.F.A.C. Synp. on control in power electronics and electrical drives, Düsseldorf (1977) pp. 593-601.

[2] Louis J. P., Goureau P. et BERGMANn C., « Comparaison des performances de servomoteurs à aimants à commutation électronique $»$, Le point en robotique, 2 (TEC. DOC. 1985) 91-100.

[3] Bose B. K., « Technology trends in microcomputer control of electrical machines », IEEE Trans. Industr. Electron. 35 (février 1988) n 1.

[4] FAdel M. et DE FoRnel B., «Control laws of a synchronous machine fed by a PWM voltage source inverter » (EPE, Aachen, RFA, October 1989).

[5] Bergmann C., Louis J. P. et Sol C., « Mathematical Models for self-controlled synchronous Motors », $2^{e}$ congrès I.M.A.C.S.-T.C.1 (Québec 1987), published in « Modelling and Simulation of Electrical Machines and Power Systems », J. Robert et D. K. Tran Eds. (Elsevier Science Publishers B. V., North-Holland, 1988) pp. 45-52.

[6] Champenois G., Mollard P. et Rognon J. P., «Synchronous servo drive: a special application ", Conf. Rec. of the 1986 IEEE-IAS Annual meeting, pp. 182-189.

[7] LeONHARD W., «Control of electrical drives » (Springer Verlag, 1985).

[8] PILlAY P. et KRISHNAN R., «Modelling, simulation, and analysis of permanent-magnet motor drives, part 1 : the permanent-magnet synchronous motor drive ", IEEE Trans. Industr. Appl. 25 (mars/avril 1989) n 2.

[9] Champenois G., Mollard P. et Rognon J. P., « Effective digital torque control systems for permanent magnets converter fed sinusoidal synchronous machine $»$, IEEE-IAS Annual meeting (1988).

[10] LE PIOUfLE B. et Louis J.-P., «Influence of the dynamics of the mechanical speed a a synchronous servomotor on its torque regulation, proposal of a robust solution » (EPE, Florence) 3 (1991) 412-417.

[11] Illic-Spong M., Marino R., Peresada S. et TAYloR D., «Feedback linearizing control of switched reluctance motors », IEEE Trans. Automatic Control 5 (1987) 371-379.

[12] DE LUCAS A. et Ulivi G., «Full linearization of induction motors via nonlinear state feedback », $26^{\mathrm{e}}$ IEEE Conf. on Decision and Control, Los Angeles (1987) 1765-1770. 
[13] Le Pioufle B., Georgiou G. et Louis J. P., « Application des commandes non linéaires pour la régulation en vitesse ou en position de la machine synchrone autopilotée », Rev. Phys. Appl. (juin 1990) 517-526.

[14] IsIDORI A., «Nonlinear control systems : an introduction » (Springer-Verlag, 1985).

[15] Georgiou G. et Le Pioufle B., « Nonlinear speed control of a synchronous servomotor with robustness » (EPE, Florence) 3 (1991) 42-48.

[16] Bergmann C., «Présentation de différentes régulations numériques de courant par la méthode de l'échantillonneur équivalent », J. Phys. III France 1 (mars 1991) 349.

[17] Reis Costa J. C., FAdel M. et DE Fornel B., « Commande numérique d'axe avec compensation du couple résistant », J. Phys. III France 1 (mars 1991) 465-479.

[18] Georgiou G., « Sur les méthodes de commande non linéaires adaptatives. Aspects échantillonnés et applications », Thèse de doctorat, Université Paris XI (1992).

[19] De LaRminat P. et Thomas Y., « Automatique des systèmes linéaires », tomes 1 et 2 (Flammarion Sciences, 1975).

[20] LuENBerger D. G., « An introduction to observers », IEEE trans. Aut. control, AC16 (1971) 596603.

[21] Dugard L. et Landau I. D., «Commande adaptative, méthodologie et applications », Hermès (1988).

[22] Le Pioufle B., Georgiou G. et Louis J.-P., « A robust totai compensation algorithm for the torque control of a synchronous servomotor », J. Phys. III France 2 (janvier 1992) 129-144.

[23] MonACo S. et NORMAND-CYRot D., « Multirate sampling and nonlinear digital control », Report DIS, Université de Rome «La Sapienza » (Italie, 1988). 